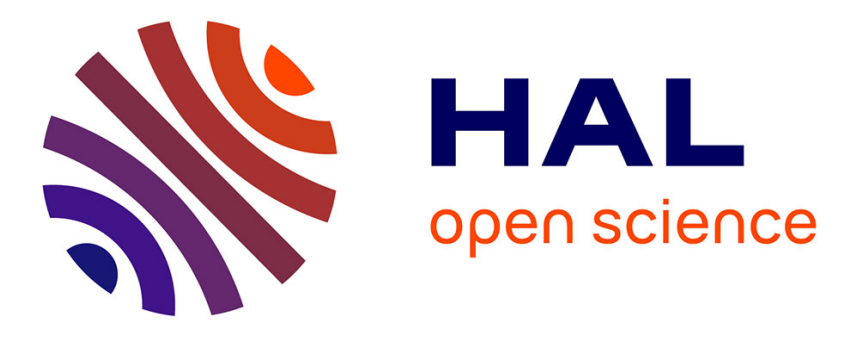

\title{
Multiple satellites in materials with complex plasmon spectra: From graphite to graphene
}

Matteo Guzzo, Joshua J. Kas, Lorenzo Sponza, Christine Giorgetti, Francesco

Sottile, Debora Pierucci, Mathieu G. Silly, Fausto Sirotti, John J. Rehr, Lucia

Reining

\section{To cite this version:}

Matteo Guzzo, Joshua J. Kas, Lorenzo Sponza, Christine Giorgetti, Francesco Sottile, et al.. Multiple satellites in materials with complex plasmon spectra: From graphite to graphene. Physical Review B: Condensed Matter and Materials Physics (1998-2015), 2014, 89, pp.085425. 10.1103/PhysRevB.89.085425 . hal-01011562

\section{HAL Id: hal-01011562 \\ https://hal-polytechnique.archives-ouvertes.fr/hal-01011562}

Submitted on 24 Jun 2014

HAL is a multi-disciplinary open access archive for the deposit and dissemination of scientific research documents, whether they are published or not. The documents may come from teaching and research institutions in France or abroad, or from public or private research centers.
L'archive ouverte pluridisciplinaire HAL, est destinée au dépôt et à la diffusion de documents scientifiques de niveau recherche, publiés ou non, émanant des établissements d'enseignement et de recherche français ou étrangers, des laboratoires publics ou privés. 


\title{
Multiple satellites in materials with complex plasmon spectra: From graphite to graphene
}

\author{
Matteo Guzzo, ${ }^{1,2,{ }^{*}}$ Joshua J. Kas, ${ }^{3}$ Lorenzo Sponza, ${ }^{1,2}$ Christine Giorgetti, ${ }^{1,2}$ Francesco Sottile,,${ }^{1,2}$ Debora Pierucci, ${ }^{4}$ \\ Mathieu G. Silly, ${ }^{4}$ Fausto Sirotti, ${ }^{4}$ John J. Rehr ${ }^{2,3}$ and Lucia Reining ${ }^{1,2, \dagger}$ \\ ${ }^{1}$ Laboratoire des Solides Irradiés, École Polytechnique, CNRS, CEA-DSM, F-91128 Palaiseau, France \\ ${ }^{2}$ European Theoretical Spectroscopy Facility (ETSF) \\ ${ }^{3}$ Department of Physics, University of Washington, Seattle, Washington 98195, USA \\ ${ }^{4}$ Synchrotron-SOLEIL, BP 48, Saint-Aubin, F91192 Gif sur Yvette CEDEX, France
}

(Received 15 February 2013; revised manuscript received 23 January 2014; published 27 February 2014)

\begin{abstract}
The photoemission spectrum of graphite is still debated. To help resolve this issue, we present photoemission measurements at high photon energy and analyze the results using a Green's function approach that takes into account the full complexity of the loss spectrum. Our measured data show multiple satellite replicas. We demonstrate that these satellites are of intrinsic origin, enhanced by extrinsic losses. The dominating satellite is due to the $\pi+\sigma$ plasmon of graphite, whereas the $\pi$ plasmon creates a tail on the high-binding energy side of the quasiparticle peak. The interplay between the two plasmons leads to energy shifts, broadening, and additional peaks in the satellite spectrum. We also predict the spectral changes in the transition from graphite towards graphene.
\end{abstract}

DOI: 10.1103/PhysRevB.89.085425

PACS number(s): 73.22.Pr, 71.45.Gm, 71.10.-w, 71.15.Qe

\section{INTRODUCTION}

Photoemission is a prominent experimental tool to access the electronic structure of materials. Angular-resolved photoelectron spectroscopy (ARPES) is frequently used to extract the band structure and quasiparticle (QP) lifetimes of a large variety of systems [1]. However, in a photoemission spectrum, besides the QP peaks and incoherent background, satellite peaks often appear. In the intrinsic spectral function, within the three-step model [2], these satellites can be understood in terms of coupling of a QP to additional excitations of the system.

Their nature is often debated. In simple metals plasmon satellite replicas are observed [3], but it is difficult to discern experimentally to which extent these are intrinsic features [4], or due to losses of the outgoing photoelectron (called extrinsic). In nickel, the satellite at $6 \mathrm{eV}$ below the QP peak cannot be explained with the plasmon spectrum, but is due to a bound hole-hole state [5,6]. Recently a satellite in doped graphene was interpreted as a plasmaron, a strongly coupled electron-plasmon excitation [7]. In $3 d$ and $4 f$ systems, satellites have been ascribed to strong electron-electron interaction within a Mott-Hubbard picture [8]. In other materials, e.g., graphite, the very existence of intrinsic satellite features has been questioned. In this system observed valence-band satellite structures have been attributed to extrinsic losses and subtracted from the measured spectrum $[9,10]$. These debates, even for supposedly simple materials, are in part due to the lack of a widely applicable theoretical approach for the description of satellites. Ab initio calculations typically ignore satellites and concentrate on QP properties, often using the $G W$ approximation for the self-energy in the framework of many-body perturbation theory [11]. In this approach the dynamically screened Coulomb interaction $W$ that multiplies

*matteo.guzzo@polytechnique.edu; present address: Physics Department, Humboldt-Universität zu Berlin, Zum Großen Windkanal 6, D-12489 Berlin, Germany.

†lucia.reining@polytechnique.fr the one-body Green's function $G$ contains plasmon excitations that may lead to satellites. However, $G W$ is believed to fail in predicting the satellite spectra [4], although it performs well for QP energies. Satellites are rarely computed, so that much of the information obtained from experiment is wasted. Our aim is to push this limit.

In the present work we focus on plasmon satellites, which occur in most materials, from metals to insulators. Plasmons lead to multiple satellites, forming a decaying series of peaks below the QP. These structures can be explained with a spectral function derived from an exponential (also referred to as cumulant) form of the one-particle Green's function, itself a solution of an electron-boson coupling model $[4,12,13]$. In the present case the plasmon plays the role of the boson, though the cumulant form is more general. The plasmon contribution has been derived in several ways, e.g., starting from $G W[4,13,14]$, or as a linear response contribution of the Hartree potential [15]. The combination of the cumulant solution with the $G W$ calculation $(\mathrm{GW}+\mathrm{C})$ and the same method with additional extrinsic and interference contributions $\left(\mathrm{GW}+\mathrm{C}^{*}\right)$ yielded excellent agreement with experiment for bulk silicon $[15,16]$. The $G W$ approximation alone, instead, gave rise to a spurious plasmaron solution in silicon, similarly to the homogeneous electron gas [17-19]. Hence, $\mathrm{GW}+\mathrm{C}^{*}$ appears to be the method of choice for plasmon satellites.

In this article we address the layered material graphite and its building block graphene. We generalize the approach used in Refs. [15,16] that was based on the use of a single plasmon pole approximation for $W$, to materials with an arbitrarily complex excitation spectrum. Our computational results are compared to new bulk sensitive photoemission data for graphite. The excellent agreement between theory and experiment allows us to address several important questions: (i) does graphite have intrinsic satellites? (ii) Does the $G W$ approximation create a spurious plasmaron also in this material? (iii) Are there any new effects in the XPS spectrum caused by the more complex plasmon spectrum, and in particular by the existence of two main plasmon peaks? Finally, it allows us to make predictions for the satellite structure in graphene. 
We start describing the experimental setup for the XPS measurements and the computational details of the calculations in Sec. II. Results are discussed in Sec. III. We analyze the shortcomings of the $G W$ approximation in graphite and discuss the absence of a plasmaron in this particular case in Sec. III B. We give the interpretation of the XPS spectrum of graphite on the basis of our $\mathrm{GW}+\mathrm{C}^{*}$ calculations in Sec. III C. Then we calculate the $\mathrm{GW}+\mathrm{C}^{*}$ spectral function of undoped graphene showing how and why this differs from its graphite counterpart in Sec. IIID. Conclusions are drawn in Sec. IV.

\section{TECHNICAL DETAILS}

\section{A. Experimental setup}

ARPES measurements were performed at the UHV photoemission station of the TEMPO beamline [20] at the SOLEIL synchrotron radiation source. Linearly polarized photons from the Apple II type Insertion Device (HU44) were selected in energy using a high resolution plane grating monochromator with a resolving power $E / \Delta E=5000$. The end-station chamber (base pressure $10^{-10}$ mbar) is equipped with a modified SCIENTA-2002 electron analyzer with a delay-line 2D detector which optimizes the detection linearity and signal/background ratio [21]. The overall energy resolution was better than $200 \mathrm{meV}$. The photon beam impinged on the sample at an angle of $43^{\circ}$, and photoelectrons were detected on an angular range of $12^{\circ}$. Highly oriented pyrolytic graphite (HOPG) was cleaved in the introduction stage of the UHV system exposing a new surface immediately before the transfer to UHV. At $800 \mathrm{eV}$ kinetic energy the Brillouin zone (BZ) is observed with an emission angle of about $8^{\circ}$. The measured photoemission map was integrated over the spectral intensity originated by one BZ. The Fermi level was obtained by measuring a clean $\mathrm{Au}(111)$ surface.

\section{B. Computational details}

We performed $a b$ initio calculations using a plane-wave basis. We used the ABINIT code for all ground-state and $G W$ calculations [22], using experimental values for lattice constants. The Brillouin zone of graphite was sampled using a $9 \times 9 \times 2$ Monkhorst-Pack grid [23], yielding 162 points in the full BZ. We have used Troullier-Martins norm-conserving pseudopotentials [24] available in the ABINIT code library. The plane-wave cutoff for the LDA ground-state calculation was fixed at $30 \mathrm{Ha}$. The full frequency dependence of the self-energy for the eight valence bands was calculated using a contour-deformation technique, and with partial self-consistency, namely on the quasiparticle energies in the Green's function ( $G W_{0}$ calculations). $W$ was calculated including 160 bands and using 997 plane waves for the wave functions. The dielectric matrix was calculated using $287 G$ vectors, 4 frequencies on the imaginary axis, and 60 frequencies on the real axis, with a maximum value fixed at $2 \mathrm{Ha}$. The self-energy was calculated using 250 bands, 1385 plane waves for the wave functions, $287 G$ vectors for the dielectric matrix, and 585 plane waves for the exchange term. The self-energy of graphite was calculated for 50 frequencies/Ha for a range of $65 \mathrm{eV}$ below the Fermi energy.

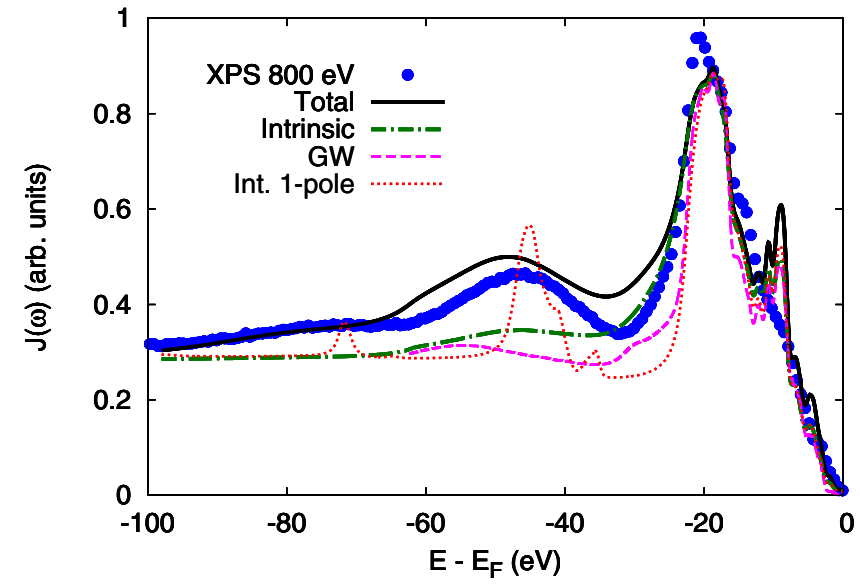

FIG. 1. (Color online) XPS spectrum of HOPG at $800 \mathrm{eV}$ photon energy. The experimental data collected at normal emission (blue dots) are compared to the spectral function $A(\omega)$ calculated from $G W$ (magenta dashed line) and from a multipole version of Eq. (1) (green dot-dashed line). On top of the latter the black solid line also includes extrinsic and interference effects. The result for $A(\omega)$ in the single plasmon pole approximation $\left(N_{p}=1\right)$ for Eq. (1) (red dotted line) is shown for comparison. All curves are scaled to match the intensity of the main QP peak at $-20 \mathrm{eV}$. All theoretical spectra contain photoabsorption cross sections, the calculated secondary electron background, and $0.4 \mathrm{eV}$ Gaussian broadening to account for finite BZ sampling and experimental resolution.

The $G W_{0}$ spectral function of graphene was calculated using equivalent parameters on a slightly different $k$-point grid $(12 \times 12 \times 1)$. We set up a supercell in real space with 38 bohrs of vacuum on the $z$ direction.

\section{RESULTS}

\section{A. Experiment}

The experimental result is shown by the blue dots in Fig. 1. The QP band structure is found between the Fermi level and $25 \mathrm{eV}$ binding energy. A tail extends down to about $-30 \mathrm{eV}$, followed by a broad satellite in the range $[-65 ;-30] \mathrm{eV}$ and a washed out structure below $-65 \mathrm{eV}$. The average distance between the main satellite and the QP region is close to the energy of the $\pi+\sigma$ plasmon $(\sim 27 \mathrm{eV})$ in the loss spectrum of graphite. There is no clear structure that one could assign to the $\pi$ plasmon ( $7 \mathrm{eV}$ in the loss spectrum). Overall there is too much broadening to draw reliable conclusions only from the data. In previous experimental studies [10,25], features beyond the QP peaks have been attributed to extrinsic background effects and subtracted from the raw data in order to obtain an "intrinsic" spectrum. Our work suggests a revision of this hypothesis, as we show below.

\section{B. Graphite- $G W$}

We first look at the results of a $G W$ calculation. The full frequency dependence of the self-energy is calculated with the ABINIT code [22], using a contour-deformation technique. The calculation involves a partial degree of self-consistency, namely on the real part of the QP energies, while $W$ is kept fixed. The total $G W$ spectral function (magenta dashed curve 

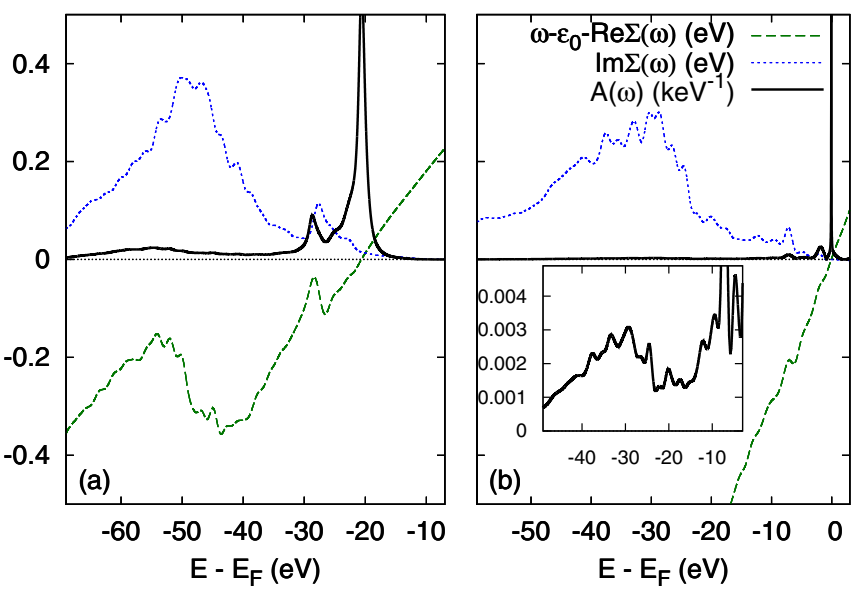

FIG. 2. (Color online) $G W$ spectral function $A(\omega)$ (solid black line) with imaginary (dotted blue line) and shifted real (dashed green line) parts of the $G W$ self-energy. (a) Lowest valence state at $k=(0.0,0.0,0.25)$. (b) Highest valence state at $k=(0.33,0.33,0.25)$. Inset: Zoom on $A(\omega)$.

in Fig. 1) well describes the QP part of the spectrum. ${ }^{1}$ Moreover, it exhibits a small bump due to the $\pi$ plasmon that may explain the tail located around $-25 \mathrm{eV}$ in the experiment and a second structure in correspondence with the first experimental satellite that can be attributed to the $\pi+\sigma$ plasmon. Overall the $G W$ result is sufficiently good for a qualitative discussion, in striking contrast to, e.g., the case of silicon.

In order to understand this point, and to exclude a simple coincidence, we analyze the $G W$ self-energy in a single plasmon-pole model. ${ }^{2}$ The correlation part of the self-energy for a hole is then approximatively $\Sigma_{c}(\omega)=\lambda /\left[\omega-\epsilon+\omega_{p}-\right.$ $i \gamma]$, with $\gamma, \omega_{p}$, and $\lambda$, respectively, the inverse lifetime, the plasmon frequency, and the strength; $\epsilon$ is the self-consistently calculated QP energy. This model describes the $G W$ selfenergy around each plasmon satellite, as one can see by qualitative comparison with the full ab initio calculation: The latter is shown in Fig. 2.

Figures 2(a) and 2(b) show the imaginary $\operatorname{Im} \Sigma$ (blue dotted line) and shifted real (green dashed line) $\omega-\varepsilon_{0}-\operatorname{Re} \Sigma(\omega)$ parts of the ab initio $G W$ self-energy for two valence states ( $\varepsilon_{0}$ is the single-particle energy). The imaginary parts exhibit two structures, associated with the $\pi$ and $\pi+\sigma$ plasmons, and the real parts have the dispersion resulting from the KramersKronig relation. The satellites in the spectral function (black solid line) are in correspondence with the local maxima $\omega_{\max }$ of the real part of the self-energy.

To first order in $\gamma$, the model yields $\omega_{\max } \approx \epsilon-\omega_{p}-\gamma$ : The $G W$ satellites are close to the expected position $\epsilon-\omega_{p}$. For larger values of $\gamma$, the shift towards larger binding energy introduced by the $G W$ approximation becomes significant. This explains the quantitative discrepancy with

\footnotetext{
${ }^{1}$ The QP peak at $-10 \mathrm{eV}$ is too sharp, which may be due to the approximate inclusion of cross-section effects and to domains in the sample.

${ }^{2}$ The energetic distance of the two plasmons in graphite is large enough to qualitatively justify such a simplification for the present discussion.
}

the experimental positions, as observed in Fig. 1. Still, the origin of the plasmon satellite remains unchanged. Instead, the model predicts that the spectrum becomes qualitatively worse when the oscillation in the real-part term is so strong that the curve crosses zero around $\omega_{\max }$ : The satellite position is then no longer determined by $\omega_{\max }$, but by the left zero-crossing point, analogously to the QP. This crossing leads to a sharp peak, the so-called plasmaron, and is a spurious effect of the $G W$ approximation [19]. The plasmaron spoils the satellite spectra of silicon [15] and the homogeneous electron gas, and is the origin for the bad reputation of $G W$ satellites. Our model shows that such a crossing occurs for $\lambda>2 \gamma \omega_{p}$ : It is favored by a large plasmon intensity and coupling, a small plasmon energy, and small broadening. This condition is not fulfilled in graphite: The $\pi$ plasmon has small intensity, and the intense $\pi+\sigma$ plasmon has large broadening. This explains why GWA can be used in graphite to analyze plasmon satellites, contrary, e.g., to the case of silicon.

However, the remaining discrepancy still does not allow definite conclusions. It is hence important to compare to the more advanced $\mathrm{GW}+\mathrm{C}^{*}$.

\section{Graphite-GW+ $\mathbf{C}^{*}$}

We have first applied the single plasmon pole model as used in Refs. $[15,16]$, reproducing the dominant $\pi+\sigma$ plasmon. The resulting intrinsic $\mathrm{GW}+\mathrm{C}$ spectral function (red dotted curve in Fig. 1) shows two satellites at -50 and $-75 \mathrm{eV}$, in very good correspondence with the experimental ones. They are however too sharp, and the tail on the QP peak is missing, contrary to the $G W$ result, evaluated with the full energy dependence of $W$. We therefore include the full $W$ also in $\mathrm{GW}+\mathrm{C}$, by adopting a multipole representation with a number of poles $N_{p}[16,26]$. The positions $\omega_{j}$ and strengths $a_{j}$ are fitted to the results of a $G W$ calculation $[15,16]$.

The final results presented here were calculated using $N_{p}=150$. However, already $N_{p}=2$ (one pole for the $\pi$ and $\pi+\sigma$ plasmon, respectively) represents the main features, and it eases the interpretation. For a given state, the spectral function $A(\omega)=1 / \pi|\operatorname{Im} G(\omega)|$ then becomes

$$
\begin{aligned}
A(\omega)= & \frac{\Gamma}{\pi} e^{-\left(a_{1}+a_{2}\right)}\left[\frac{1}{(\omega-\epsilon)^{2}+\Gamma^{2}}+\frac{a_{1}}{\left(\omega-\epsilon+\omega_{1}\right)^{2}+\Gamma^{2}}\right. \\
& +\frac{a_{2}}{\left(\omega-\epsilon+\omega_{2}\right)^{2}+\Gamma^{2}}+\frac{1}{2} \frac{a_{1}^{2}}{\left(\omega-\epsilon+2 \omega_{1}\right)^{2}+\Gamma^{2}} \\
& +\frac{1}{2} \frac{a_{2}^{2}}{\left(\omega-\epsilon+2 \omega_{2}\right)^{2}+\Gamma^{2}} \\
& \left.+\frac{a_{1} a_{2}}{\left(\omega-\epsilon+\omega_{1}+\omega_{2}\right)^{2}+\Gamma^{2}}+\cdots\right]
\end{aligned}
$$

where $\epsilon+i \Gamma$ is the QP energy including lifetime broadening. The $a_{j}$ and $\omega_{j}$ are the intensities and frequencies, respectively, associated with the plasmons. The spectral function shows a peak at the QP energy $\epsilon$, followed by a series of satellite peaks. Their distance from the QP peak corresponds to sums of plasmon pole energies; for a peak of order $n$, one finds peaks at a distance of $n$ (equal or different) plasmon energies from $\epsilon$. The weight of each peak is the product of the corresponding $a_{j}$ and a prefactor due to the expansion. 


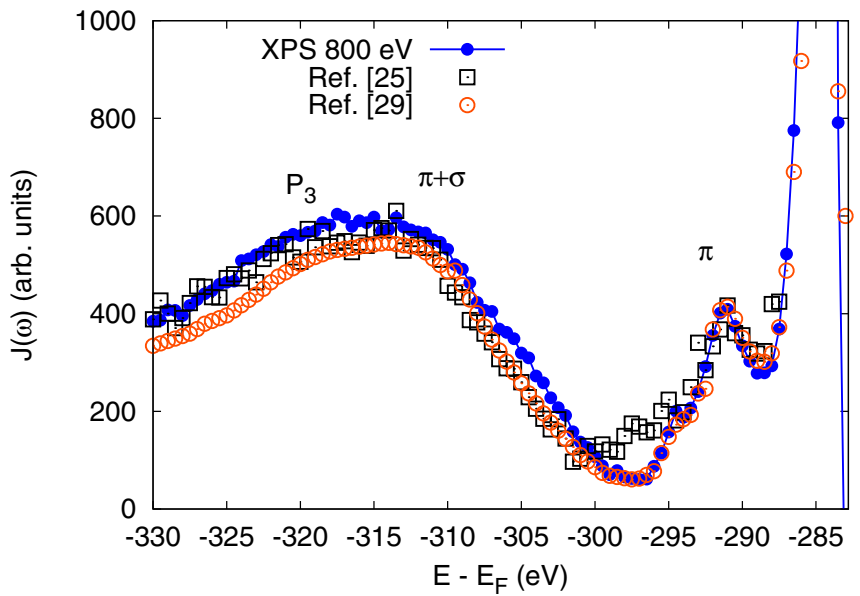

FIG. 3. (Color online) C $1 s$ XPS spectrum of HOPG at $800 \mathrm{eV}$ photon energy as measured in the present work (blue line with points), Ref. [25] (black squares), and in Ref. [27] (orange circles). Signatures of the $\pi$ and $\pi+\sigma$ plasmons are clearly visible, as well as the feature termed $P_{3}$ in Refs. [25,27].

Equation (1) yields a first decaying series of plasmon satellites at a distance from the QP peak of multiples of $\omega_{1}$, and a second series from $\omega_{2}$. Moreover, a new series of multiple satellites results from the sum of multiples of $\omega_{1}$ and $\omega_{2}$, with the smallest being $\omega_{1}+\omega_{2}$; the intensity of this peak is proportional to $a_{1} a_{2}$. It is significantly more intense than the smaller second-order peak of the two because $a_{1} a_{2}>a_{1}^{2} / 2$ for $a_{1}<2 a_{2}$. Higher orders form additional structures centered at $n \omega_{1}+m \omega_{2}$ with intensity $n a_{1} m a_{2}$. Since these mixed peaks have no one-to-one correspondence with the loss spectrum, their interpretation needs the support of theory.

The resulting spectral function $A(\omega)$ (green dot-dashed curve in Fig. 1), calculated for $N_{p}=150$, shows the closest agreement in peak positions with the experimental result, much better than both the $G W$ and the single pole ones. The tail below the QP region is well reproduced, the first satellite is in the correct position, and there is significant broadening as found in experiment. The second satellite at $-75 \mathrm{eV}$ is washed out. Contrary to a previous hypothesis, at least the first-order part of the satellite spectrum of graphite has hence a significant intrinsic contribution. To further add extrinsic and interference contributions, following $[2,16]$, we renormalize the coefficients $a_{j}$ and add a lifetime broadening to the plasmon energies. All parameters are calculated, not fitted, for $800 \mathrm{eV}$ photon energy. We also include in all spectra the calculated secondary electron background and cross sections as described in Ref. [16]. The $\mathrm{GW}+\mathrm{C}^{*}$ result (black continuous curve in Fig. 1) is in excellent agreement with experiment, confirming the validity of the approach and the resulting analysis.

Satellites are resolved especially well in core-level spectra. Figure 3 shows our measured carbon $1 s$ spectrum together with previous experimental results [25,27]. Both the signature of the $\pi$ plasmon and the presence of at least one additional structure on the $\pi+\sigma$ plasmon satellite are clearly visible. The additional structure has been termed $P_{3}$, and measured in various carbon based materials [25] (see also, e.g., [27]), but could never be explained to our knowledge. However, for all measured materials it appears to be situated approximatively

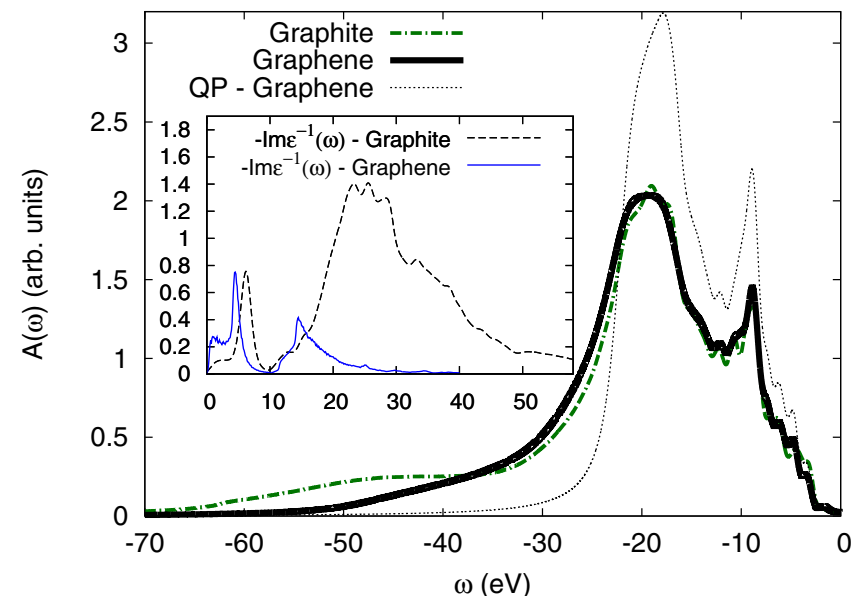

FIG. 4. (Color online) Comparison of graphite (dot-dashed green line) and graphene (solid black line) spectral functions using $\mathrm{GW}+\mathrm{C}$. The QP part of graphene (dotted black line) is reported for comparison. The QP and satellite tail at $-30 \mathrm{eV}$ do not change going from graphite to graphene, while most of the satellite weight below $-40 \mathrm{eV}$ disappears. This change stems from the differences in the loss functions. Inset: The calculated loss function $-\operatorname{Im}\left[\varepsilon^{-1}\right]$ (arbitrary units vs eV) of graphite (dashed black line) is compared to graphene (solid blue line). The curves are scaled to match the intensity of the $\pi$ plasmon peak.

at the sum of the materials-dependent energies of the first two main plasmon peaks [25]. We can therefore identify it with the the $\omega_{1}+\omega_{2}$ peak predicted by our Eq. (1).

The superposition of states in the valence broadens the spectrum, so that the $\pi$ plasmon satellite close to the QP only shows up as a tail. Moreover, the $\omega_{1}+\omega_{2}$ peak on the $\pi+\sigma$ satellite cannot be resolved. However, the position of the center of mass of the $\pi+\sigma$ satellite in the full spectrum in Fig. 1 shows a shift of $\sim 2 \mathrm{eV}$ to higher binding energy as compared to the single pole calculation, which can be ascribed to the $\omega_{1}+$ $\omega_{2}$ contribution (see the Appendix for quantitative details). We can conclude that this mixed term leads in graphite to a shift of the main valence plasmon satellite and to an additional broadening. It contributes to the excellent agreement between our final result (black continuous curve) and experiment. Thus the approach allows us to fully describe and understand the XPS of graphite and to conclude the long standing debate about the nature of observed incoherent structures.

\section{Graphene}

These convincing results allow us to extend the calculations to graphene. It is important to produce benchmark results for ideal freestanding graphene, because experimental photoemission results are obtained on substrates, which complicates the interpretation. The transition from graphite to graphene, from three to two dimensions, is also interesting from a purely fundamental point of view.

Figure 4 shows the result of our calculations. The green dot-dashed curve is the graphite intrinsic spectral function (same as Fig. 1, without secondary-electron background). The black continuous curve is the $\mathrm{GW}+\mathrm{C}$ result for graphene. In the QP region the two materials are extremely similar: Indeed, 


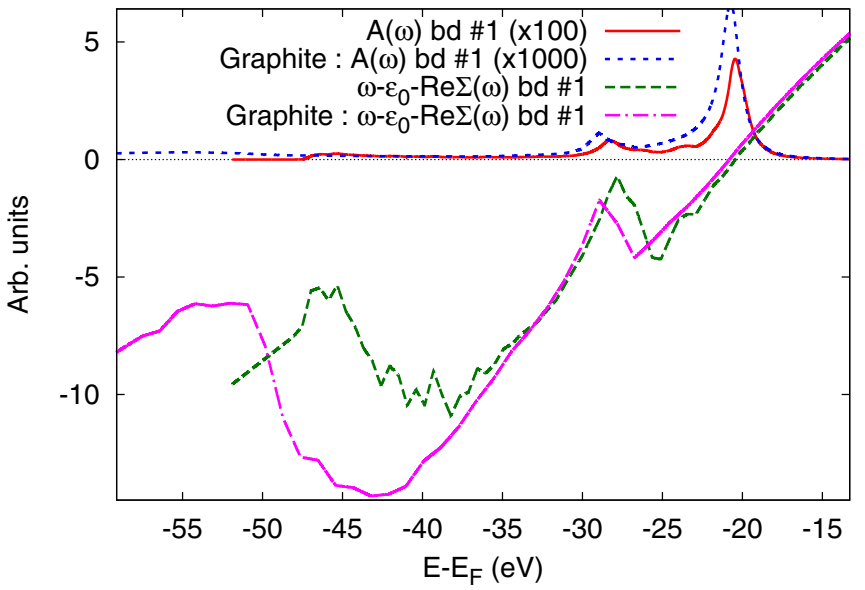

FIG. 5. (Color online) $\Gamma$-point $G W_{0}$ spectral function $A(\omega)$ for graphene (solid red line) and graphite (dotted blue line), along with the shifted real-part term of the self-energy for the lowest valence band of graphene (dashed green line) and graphite (dot-dashed magenta line), respectively.

the charge density on the graphene layers changes only a little when the sheets are brought together to form graphite, with weak hybridization and a similar band structure. However, satellites are due to plasmon excitations which involve the long-range Coulomb interaction. This long-range interaction "detects" the presence of additional layers even at far distances. The plasmon spectra of graphite and graphene are therefore completely different (see inset in Fig. 4). In particular the $\pi+\sigma$ plasmon in graphene disappears, with only a small contribution remaining at much lower energies $(\sim 15 \mathrm{eV})$, which explains the fading of the corresponding satellite in photoemission. The $\pi$ plasmon is more stable and indeed, the corresponding tail on the QP peak remains intact. As our work confirms, this tail is an intrinsic feature of graphite and graphene.

For more detailed comparison, we also look at single bands including the corresponding self-energy contributions. Plasmaron solutions are defined as crossing of the $\omega-\varepsilon_{0}-$ $\operatorname{Re} \Sigma(\omega)$ curve with the horizontal axis, at higher binding energies than the QP peak. Figure 5 shows that there are no plasmaron solutions either in graphite or graphene. The behavior of the self-energy is similar in the two cases, as it is shown in Fig. 5. However, the shifted real-part term in graphite has a relatively steep slope stemming from the $\pi+\sigma$ plasmon, which leads to significant screening of the $\pi$ plasmon structure. In graphene instead, the $\pi+\sigma$ plasmon is strongly suppressed, hence the structure in the real-part term due to the $\pi$ plasmon is closer to zero. Still, our $G W$ calculation shows no plasmaron solution for undoped graphene. Instead, doping creates carriers that lead to additional plasmon excitations at very low energies (small $\omega_{p}$ ). The observed satellite lines [7] related to these excitations are sharp (small $\gamma)$. In this parameter range our model predicts that a $G W$ calculation yields a spurious plasmaron, which can be cured by $\mathrm{GW}+\mathrm{C}^{*}$, as it has been demonstrated by recent ab initio calculations [28].

\section{CONCLUSION}

We have conducted a joint experimental and theoretical study to understand plasmon satellites in materials very different from the homogeneous electron gas, using graphite as a prototype case. We have extended the calculation of the photoemission spectra based on $\mathrm{GW}+\mathrm{C}^{*}$ to the case of a material with more than one dominant collective excitation. The comparison to state-of-the art photoemission data over a wide range of binding energies confirms the predictive power of our approach. We can explain in detail the photoemission spectrum of graphite, and in particular demonstrate that it contains an intrinsic satellite contribution caused by the $\pi+\sigma$ plasmon, while the $\pi$ plasmon leads to a tail on the QP peak. The interplay of the two plasmons causes an additional broadening and shift of the main satellite, and explains additional structures in cases of better resolution, e.g., core levels. We also discuss the problem of the spurious plasmaron in $G W$ calculations, showing that, and why, the problem does not occur in undoped graphite, nor in undoped graphene, and why additional excitations due to doping create a more critical scenario.

\section{ACKNOWLEDGMENTS}

We acknowledge support by ANR (NT09-610745) and the RTRA Triangle de la Physique. J.J.R. and J.J.K. are also supported in part by DOE BES Grant DE-FG03-97ER45623 and DOE CMCSN. Computer time was granted by GENCI (544).

\section{APPENDIX: PEAK-SHIFTING EFFECT IN SPECTRA WITH MULTIPLE SATELLITES}

Here we describe in detail how one can estimate the shift of the first main satellite in the photoemission spectrum of graphite (roughly located at $-50 \mathrm{eV}$ ) due to the two-plasmon structure of the energy-loss spectrum of graphite. The spectral function for $N_{p}=2$ is given by (1). Our aim is to compare the position of the main satellite stemming from the $\sigma+\pi$ plasmon $\left(\omega_{2}\right.$ with weight $\left.a_{2}\right)$ to the average position that is obtained when also the first mixed satellite $\left(\omega_{1}+\omega_{2}\right.$ with weight $a_{1} a_{2}$ ) is taken into account.

The calculated parameters concerning the intrinsic (superscript int) and total, i.e., intrinsic plus extrinsic and interference (superscript eff) contributions at $\omega_{1}$ and $\omega_{2}$ are

\begin{tabular}{lcc}
\hline \hline$\omega_{j}(\mathrm{eV})$ & $a_{j}^{\mathrm{int}}$ & $a_{j}^{\mathrm{eff}}$ \\
\hline 7.78 & 0.205 & 0.331 \\
29.85 & 0.156 & 0.872 \\
\hline \hline
\end{tabular}

hence for $\omega_{1}+\omega_{2}=37.63$ we have weights $a_{1}^{\text {int }} a_{2}^{\text {int }}=0.032$ and $a_{1}^{\text {eff }} a_{2}^{\text {eff }}=0.289$. These values are averaged over the Brillouin zone. To first approximation the center of mass of the structure composed by the $\pi+\sigma$ and the first mixed satellite is

$$
\bar{\omega}=\frac{a_{1} a_{2}\left(\omega_{1}+\omega_{2}\right)+a_{2} \omega_{2}}{a_{1} a_{2}+a_{2}} .
$$

The deviation from the main $\sigma+\pi$ peak is defined as $\Delta \omega=$ $\left|\bar{\omega}-\omega_{2}\right|$. We obtain

$$
\Delta \omega^{\text {int }}=1.3, \Delta \omega^{\text {eff }}=1.9
$$

for the purely intrinsic and for the total shift, respectively. 
[1] A. Damascelli, Z. Hussain, and Z.-X. Shen, Rev. Mod. Phys. 75, 473 (2003).

[2] L. Hedin, J. Michiels, and J. Inglesfield, Phys. Rev. B 58, 15565 (1998).

[3] P. Steiner, H. Höchst, and S. Hüfner, Topics in Applied Physics: Photoemission in Solids II (Springer, Heidelberg, 1979).

[4] F. Aryasetiawan, L. Hedin, and K. Karlsson, Phys. Rev. Lett. 77, 2268 (1996).

[5] C. Guillot, Y. Ballu, J. Paigné, J. Lecante, K. P. Jain, P. Thiry, R. Pinchaux, Y. Pétroff, and L. M. Falicov, Phys. Rev. Lett. 39, 1632 (1977).

[6] A. Liebsch, Phys. Rev. Lett. 43, 1431 (1979).

[7] A. Bostwick, F. Speck, T. Seyller, K. Horn, M. Polini, R. Asgari, A. H. MacDonald, and E. Rotenberg, Science 328, 999 (2010).

[8] A. K. McMahan, K. Held, and R. T. Scalettar, Phys. Rev. B 67, 075108 (2003).

[9] M. Vos, A. S. Kheifets, E. Weigold, and F. Aryasetiawan, Phys. Rev. B 63, 033108 (2001).

[10] T. Sattler, T. Tschentscher, J. R. Schneider, M. Vos, A. S. Kheifets, D. R. Lun, E. Weigold, G. Dollinger, H. Bross, and F. Bell, Phys. Rev. B 63, 155204 (2001).

[11] L. Hedin, Phys. Rev. 139, A796 (1965).

[12] D. C. Langreth, Phys. Rev. B 1, 471 (1970).

[13] L. Hedin, J. Phys.: Condens. Matter 11, R489 (1999).

[14] C.-O. Almbladh and L. Hedin, Handbook on Synchrotron Radiation (North-Holland, Amsterdam, 1983).

[15] M. Guzzo, G. Lani, F. Sottile, P. Romaniello, M. Gatti, J. J. Kas, J. J. Rehr, M. G. Silly, F. Sirotti, and L. Reining, Phys. Rev. Lett. 107, 166401 (2011).
[16] M. Guzzo, J. Kas, F. Sottile, M. Silly, F. Sirotti, J. Rehr, and L. Reining, Eur. Phys. J. B 85, 324 (2012).

[17] L. Hedin, B. Lundqvist, and S. Lundqvist, Solid State Commun. 5, 237 (1967).

[18] B. I. Lundqvist, Phys. Kondens. Mater. 9, 236 (1969).

[19] C. Blomberg and B. Bergersen, Can. J. Phys. 50, 2286 (1972).

[20] F. Polack, M. Silly, C. Chauvet, B. Lagarde, N. Bergeard, M. Izquierdo, O. Chubar, D. Krizmancic, M. Ribbens, J.-P. Duval, C. Basset, S. Kubsky, and F. Sirotti, AIP Conf. Proc. 1234, 185 (2010).

[21] N. Bergeard, M. Silly, D. Krizmancic, C. Chauvet, M. Guzzo, J. Ricaud, M. Izquierdo, L. Stebel, P. Pittana, R. Sergo, G. Cautero, G. Dufour, F. Rochet, and F. Sirotti, J. Synch. Rad. 18, 245 (2011).

[22] X. Gonze, G.-M. Rignanese, M. Verstraete, J.-M. Beuken, Y. Pouillon, R. Caracas, F. Jollet, M. Torrent, G. Zerah, M. Mikami, P. Ghosez, M. Veithen, J.-Y. Raty, V. Olevano, F. Bruneval, L. Reining, R. Godby, G. Onida, D. Hamann, and D. Allan, Z. Kristallogr. 220, 558 (2009).

[23] H. J. Monkhorst and J. D. Pack, Phys. Rev. B 13, 5188 (1976).

[24] N. Troullier and J. L. Martins, Phys. Rev. B 43, 1993 (1991).

[25] F. R. McFeely, S. P. Kowalczyk, L. Ley, R. G. Cavell, R. A. Pollak, and D. A. Shirley, Phys. Rev. B 9, 5268 (1974).

[26] J. J. Kas, A. P. Sorini, M. P. Prange, L. W. Cambell, J. A. Soininen, and J. J. Rehr, Phys. Rev. B 76, 195116 (2007).

[27] J. Leiro, M. Heinonen, T. Laiho, and I. Batirev, J. Electron Spectrosc. Relat. Phenom. 128, 205 (2003).

[28] J. Lischner, D. Vigil-Fowler, and S. G. Louie, Phys. Rev. Lett. 110, 146801 (2013). 